drome (AECG 2002 and ACR/EULAR 2017) did not include major salivary glands ultrasononography (SGUS).

Objectives: The UTOPIA study was undertaken to determine if and how SGUS may improve the ACR/EULAR criteria.

Methods: Twenty four international experts in pSS evaluated on an internetsecure relational database 512 randomly realistic vignettes derived from 150 patients with suspected pSS included in the french DiapSS cohort. Each vignette contained sections on "history" (duration of the symptoms, gender, age), clinical symptoms (dry mouth, dry eyes and systemic manifestations), results of the SGUS evaluation (score $>$ ou $<$ to 2), and results of the major tests to diagnose pSS (Schirmer's test, ocular staining score (OSS), salivary flow, focus score on salivary biopsy, presence of anti-SSA antibodies). Each expert had to score the diagnosis of pSS as absent, unlikely, likely or present for 64 vignettes. Each vignette was evaluated by 3 experts. Diagnosis of pSS was obtained when at least 2 of 3 considered it as likely or present. Univariate and multivariate analysis (Wald test) were performed to evaluate the association between the SGUS criteria, the ACR/EULAR criteria and its different individual items with the diagnosis of pSS as defined by the experts. Data were then replicated on independent cohorts of suspicion of pSS.

Results: Univariate and multivariate analyses confirmed that ACR/EULAR criteria and SGUS were independently associated with the diagnosis of pSS. Disease duration, OSS and ocular dryness were not associated with the diagnosis of pSS. Only 6 variables were selected by logistic regression analysis: presence of anti-SSA (weight:4), focus score (weight:3), SGUS (weight:2), Schirmer's test (weight:1), dry mouth (weight:1) and salivary flow rate (weight:1). According to ROC curve analysis, a score of $\geq 5$ had $96 \%$ Se and $84 \%$ Sp, compared with $90 \%$ Se and $84 \%$ Sp for the ACR/EULAR criteria. The corrected C statistic (AUC) for the new weighted score was 0.98 .

Conclusions: Inclusion of the SGUS item in the ACR/EULAR criteria improves their diagnostic performance.

Disclosure of Interest: S. Jousse-Joulin: None declared, F. Gatineau: None declared, C. Baldini: None declared, S. Arends: None declared, F. Barone: None declared, A. Baer: None declared, H. Bootsma: None declared, S. Bowman: None declared, P. Brito-Zeron: None declared, D. Cornec: None declared, T. Dorner: None declared, S. De Vita: None declared, B. Fisher: None declared, D. Hammenfors: None declared, M. Jonsson: None declared, X. Mariette: None declared, V. Milic: None declared, T. Nakamura: None declared, W.-F. Ng: None declared, E. Nowak: None declared, A. Rasmussen: None declared, R. Seror: None declared, C. Shiboski: None declared, T. Nakamura: None declared, M. Ramos-Casals: None declared, A. Vissink: None declared, A. saraux: None declared, V. Devauchelle-Pensec Grant/research support from: ROCHE-CHUGAI DOI: 10.1136/annrheumdis-2017-eular.3204

\section{OP0041 ALL-CAUSE, CARDIOVASCULAR AND MALIGNANCY RELATED MORTALITY IN SYSTEMIC LUPUS ERYTHEMATOSUS (SLE): A POPULATION-BASED STUDY}

B. Moghaddam ${ }^{1}$, J.A. Avina-Zubieta ${ }^{2,3}$, E.C. Sayre ${ }^{2} \cdot{ }^{1}$ Division of Medicine, University of British Columbia, Vancouver; ${ }^{2}$ Arthritis Research Canada, Richmond; ${ }^{3}$ Division of Rheumatology, University of British Columbia, Vancouver, Canada

Background: SLE is associated with increased risk of overall mortality; however morality trends due to specific causes including cardiovascular disease (CVD), malignancies or other causes are largely unknown.

Objectives: Our objective was to assess trends in cause-specific mortality among SLE patients between January 1, 1997 and December 31, 2012 in a general population-based context.

Methods: We conducted a population-based matched cohort on SLE patients diagnosed between January 1,1997 and December 31,2012 using an administrative health database from the province of British Columbia, Canada. We identified all incident cases of SLE and up to 10 non-SLE controls matched on sex, age, and calendar year of study entry. The cohort was divided into two cohorts based on year of SLE diagnosis (1997-2004 and 2005-2012). All-cause mortality and cause-specific incidence of death rates (IR) were calculated. Cox proportional hazard regression models were used to estimate the mortality hazard ratios (HR), adjusting for possible confounders (i.e. Charlson Comorbidity Index, number of outpatient visits, hospitalization, cardiovascular medications, glucocorticoids and NSAIDs at baseline).
Results: 4238 SLE and 42380 matched controls were studied. SLE patients had significantly increased all-cause mortality with HR $1.29(95 \% \mathrm{Cl}, 1.15-1.46)$ and increased cause-specific mortality from CVD and other causes with HRs of $1.43(95 \% \mathrm{Cl}, 1.15-1.79)$ and $1.74(95 \% \mathrm{Cl}, 1.46-2.09)$, respectively. The cohorts did not differ in the rate of death from malignancy. SLE patients had an approximately 2-fold increase in death from other causes in both early (HR 1.86 (95\% Cl 1.33-2.60)) and recent cohorts (HR $1.90(95 \% \mathrm{Cl} 1.42-2.56))$. There was no significant improvement in all-cause and cause-specific mortality trends between the two cohorts.

Conclusions: This study demonstrates that despite advances in therapy with novel biologic agents, there are no significant differences in all-cause and CVD mortality from SLE between early and recent cohorts. Death from other causes, which includes a composite of death related to for example renal disease and infections, remains high suggesting areas for future targeted research and therapy. Disclosure of Interest: None declared

DOI: 10.1136/annrheumdis-2017-eular.4451

\section{OP0042 BACTEREMIA IN SYSTEMIC LUPUS ERYTHEMATOSUS PATIENTS FROM RELESSER REGISTRY: RISK FACTORS, CLINICAL AND MICROBIOLOGICAL CHARACTERISTICS AND OUTCOMES}

I. Rua-Figueroa ${ }^{1}$, J. López-Longo ${ }^{2}$, M. Galindo-Izquierdo ${ }^{3}$, V. Del Campo ${ }^{4}$, J. De la Torre Cisneros ${ }^{5}$, E. Uriarte ${ }^{6}$, P. Vela $^{7}$, E. Tomero ${ }^{8}$, J. Pego-Reigosa ${ }^{4}$ ${ }^{1}$ Rheumatology, Hospital Dr. Negrin, Las Palmas GC; ${ }^{2}$ Gregorio Marañón Hospital; ${ }^{3}$ Doce de Octubre Hospital, Madrid (Spain), Madrid; ${ }^{4}$ Hospital Complex of Vigo and IISGS, Vigo; ${ }^{5}$ Reina Sofía Hospital, Córdoba; ${ }^{6}$ Donosti Hospital, Guipúzcoa; ${ }^{7}$ Alicante G Hospital, Alicante; ${ }^{8}$ La Princesa Hospital, Madrid, Spain

Background: According to RELESSER (Spanish Society of Rheumatology Lupus Registry) data, bacteremia is the main cause of death by infection in systemic lupus erythematosus (SLE). However, the available information about this severe infection in SLE patients remains scarce.

Methods: Retrospective, nested case-control study of SLE patients (ACR97 criteria) with at least one bacteremic episode and random controls from RELESSER Registry. Descriptive, bivariate and multivariate analysis (logistic regression)

Results: 114 bacteremic episodes in 83 patients were recorded. Incidence rate: $2,7 / 1,000$ patient-years ( $n$ total: 3658 ). At the time of the bacteremia: median age: 40.5 (8-90) years, $88.6 \%$ female, disease duration: 9.7 (IR16.7), median SELENA-SLEDAI: 4 (IR8), $66 \%$ with severe flare (SFI criteria), active nephritis: 16.7\%, median SLICC/ACR DI: 3 (IR4), any comorbidity: 64\% (McCabeJackson criteria: $28.1 \%$ rapidly or ultimately fatal), more frequently renal failure $(15.8 \%)$ or diabetes $(11.4 \%)$. SLE treatment at the time of bacteraemia: $88.6 \%$ corticosteroids $(68,6 \%>10 \mathrm{mg} /$ day), $57 \%$ immunosuppressors (mycophenolate $17.5 \%$ and cyclophosphamide $12.3 \%$ ), $27 \%$ antimalarials. $44.7 \%$ suffered invasive procedures, more frequently intravascular catheter $(24.6 \%)$. The bacteremia was nosocomial in $35.1 \%$ and the source was more frequently urinary $(27.2 \%)$. $64 \%$ developed systemic inflammatory response syndrome and $35 \%$ needed intensive care unit admission, with multiorganic failure in $22.8 \%$. The most frequent microorganism was E.coli (29.8\%) followed by Staphylococcus aureus (16.7\%) (22\% methicillin-resistant) and Salmonella spp. (10.5\%). $16 \%$ of the gramnegative enteric bacilli were extended-spectrum b-lactamase positive. $17.5 \%$ were multidrug resistant. $68,4 \%$ started the antibiotherapy before blood culture results, resulting finally active in susceptibility testing in 56 cases $(71.8 \%)$, indicating an appropriate empirical antibiotic therapy in $49 \%$. The bacteremia-related mortality was $14 \%$. The risk of death was higher in patients with severe sepsis (Pitt index >8) (OR: 13 (IC95\%: 3.71-45.17). The bacteremia was recurrent in $26.3 \%$. Associations with bacteremia in bivariate analysis (114 bacteremias vs 688 controls) are shown in Table 1 . Antimalarials were protective. In the multivariate analysis (adjusted for disease duration), only elevated creatinine (OR $1.31(95 \% \mathrm{Cl}$ 1.01-1.70), $p=0.045$ ), diabetes (OR 6.01 (95\% Cl 2.26-15.95), $p=0.000$ ), cancer (OR 5.32 (95\% Cl 2.23-12.70), $\mathrm{p}=0.000$ ), immunosuppressors (OR $6.35(95 \%$ Cl 3.42-11.77), $\mathrm{p}=0.000$ ), cyclophosphamide (OR 9.37 (95\% Cl 5.12-17.14), $\mathrm{p}=0.000)$ and SLICC/ACR DI (OR $1.65(95 \% \mathrm{Cl} 1.31-2.09), \mathrm{p}=0.000)$ remained statistically significant.

Conclusions: Bacteremia occurred mostly in active SLE, frequently in the context of a severe flare. Gram negative bacilli predominated, with high rate of multidrug resistance. The empiric treatment was inappropriate in a half of the cases. The

Abstract OP0041 - Table 1. Overall and cause-specific mortality in SLE patients compared to general population; HR, hazard ratio

\begin{tabular}{|c|c|c|c|c|c|c|c|c|c|}
\hline \multirow[t]{2}{*}{ Cohorts } & & \multicolumn{2}{|c|}{ All-Cause Deaths } & \multicolumn{2}{|c|}{ CVD Deaths } & \multicolumn{2}{|c|}{ Malignancy Deaths } & \multicolumn{2}{|c|}{ Other Causes } \\
\hline & & Deaths & $\mathrm{HR}(95 \% \mathrm{Cl})$ & Deaths & $\mathrm{HR}(95 \% \mathrm{Cl})$ & Deaths & $\mathrm{HR}(95 \% \mathrm{Cl})$ & Deaths & $\mathrm{HR}(95 \% \mathrm{Cl})$ \\
\hline \multirow[t]{2}{*}{ Overall Cohort } & $\operatorname{SLE}(n=4238)$ & 411 & $1.29(1.15-1.46)$ & 104 & $1.43(1.15-1.79)$ & 95 & $0.80(0.63-1.00)$ & 212 & $1.74(1.46-2.09)$ \\
\hline & Non-SLE $(n=42380)$ & 2226 & 1 & 622 & 1 & 795 & 1 & 809 & 1 \\
\hline \multirow[t]{2}{*}{ Female Cohort } & $\operatorname{SLE}(n=3643)$ & 323 & $1.34(1.17-1.54)$ & 75 & $1.40(1.08-1.81)$ & 77 & $0.81(0.62-1.05)$ & 171 & $2.05(1.68-2.50)$ \\
\hline & Non-SLE $(n=36430)$ & 1697 & 1 & 469 & 1 & 618 & 1 & 610 & 1 \\
\hline \multirow[t]{2}{*}{ Male Cohort } & SLE $(n=595)$ & 88 & $1.06(0.81-1.39)$ & 29 & $1.73(1.10-2.71)$ & 18 & $0.76(0.44-1.30)$ & 41 & $1.06(0.70-1.62)$ \\
\hline & Non-SLE $(\mathrm{n}=5950)$ & 529 & 1 & 153 & 1 & 177 & 1 & 199 & 1 \\
\hline \multirow{2}{*}{ Early Cohort (1997-2004) } & $\operatorname{SLE}(n=1678)$ & 98 & $1.20(0.96-1.51)$ & 25 & $1.18(0.76-1.82)$ & 22 & $0.68(0.42-1.09)$ & 51 & $1.86(1.33-2.60)$ \\
\hline & Non-SLE $(n=16780)$ & 508 & 1 & 163 & 1 & 173 & 1 & 172 & 1 \\
\hline \multirow[t]{2}{*}{ Recent Cohort (2005-2012) } & SLE $(n=2560)$ & 137 & $1.13(0.92-1.39)$ & 25 & $0.89(0.57-1.39)$ & 33 & $0.66(0.45-0.98)$ & 79 & $1.90(1.42-2.56)$ \\
\hline & Non-SLE $(n=25600)$ & 622 & 1 & 179 & 1 & 260 & 1 & 223 & 1 \\
\hline
\end{tabular}

